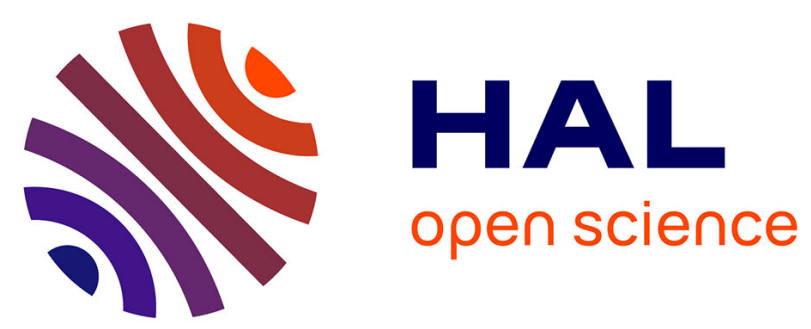

\title{
MODÉLISATION, AU MOYEN DU SIGNAL ANALYTIQUE, DE L'EFFET DOPPLER ULTRASONORE PRODUIT PAR UNE CIBLE DONT LA VITESSE DÉPEND DU TEMPS
}

\author{
G. Gimenez, C. Cachard, D. Vray, F. Denis
}

\section{To cite this version:}

G. Gimenez, C. Cachard, D. Vray, F. Denis. MODÉlisATION, AU MOYEN DU SIGNAL ANALYTIQUE, DE L'EFFET DOPPLER ULTRASONORE PRODUIT PAR UNE CIBLE DONT LA VITESSE DÉPEND DU TEMPS. Journal de Physique Colloques, 1990, 51 (C2), pp.C2-37-C2-40. 10.1051/jphyscol:1990209 . jpa-00230378

\section{HAL Id: jpa-00230378 https://hal.science/jpa-00230378}

Submitted on 1 Jan 1990

HAL is a multi-disciplinary open access archive for the deposit and dissemination of scientific research documents, whether they are published or not. The documents may come from teaching and research institutions in France or abroad, or from public or private research centers.
L'archive ouverte pluridisciplinaire HAL, est destinée au dépôt et à la diffusion de documents scientifiques de niveau recherche, publiés ou non, émanant des établissements d'enseignement et de recherche français ou étrangers, des laboratoires publics ou privés. 
COLLOQUE DE PHYSIQUE

Colloque C2, supplément au $\mathrm{n}^{\circ} 2$, Tome 51 , Février 1990

ler Congrès Français d'Acoustique 1990

MODÉLISATION, AU MOYEN DU SIGNAL ANALYTIQUE, DE L'EFFET DOPPLER ULTRASONORE PRODUIT PAR UNE CIBLE DONT LA VITESSE DEPEND DU TEMPS

G. GIMENEZ, C. CACHARD, D. VRAY et F. DENIS

Laboratoire "Traitement du Signal et Ultrasons", INSA Bât. 502, 20

Avenue Albert Einstein, F-69621 Villeurbanne Cedex, France

Résumé : Le concept de fréquence instantanée est utilisé pour décrire l'effet Doppler produit par une cible en mouvement rectiligne uniforme, le transducteur émetteur-récepteur n'étant pas situé sur la trajectoire. La vitesse radiale dépendant du temps, la fréquence Doppler, fréquence de l'enveloppe complexe associée au signal reçu, dépend également du temps. La prise en compte de l'amplitude des signaux permet alors de simuler la situation expérimentale.

Abstract : The instantaneous frequency concept is used to describe the Doppler effect due to a target moving with a constant velocity on a rectilinear trajectory, the transmitter receiver beeing off this trajectory. The radial velocity beeing time-dependant, so is the Doppler frequency. This frequency is the frequency of the complex envelope of the received signal. Assuming spherical wave propagation it is possible to predict the time-evolution of the received complex envelope for the investigated Doppler situation.

\section{1 - INTRODUCTION}

L'effet Doppler est souvent utilisé pour mesurer la vitesse d'une cible. Quand cette vitesse est constante durant la traversée du champ de mesure, la fréquence Doppler l'est aussi et l'interprétation du résultat de la mesure se fait sans problème. Dès que le module et/ou la direction de la vitesse varie, on ne peut extraire du signal qu'une pseudo-période qui varie d'un cycle au suivant. La notion de fréquence instantanée, liée au concept de signal analytique permet alors de décrire le phénomène de manière satisfaisante $[1,2]$. Pour l'obtenir il faut accéder non seulement au signal lui-même mais aussi au signal en quadrature, [3, 4].

Dans ce travail on évalue le signal analytique et les grandeurs associées : fréquence instantanée, enveloppe complexe pour un signal retardé par rapport à un signal de référence. Cela permet alors de définir la fréquence Doppler. On examine alors le cas de l'effet Doppler produit par une cible en mouvement rectiligne uniforme, le transducteur émetteur-récepteur n'étant pas situé sur sa trajectoire. On suppose enfin que l'onde émise et l'onde réfléchie sont sphériques pour simuler cette situation.

\section{2 - ETUDE D'UN SIGNAL RETARDE}

Soit $\underline{\underline{\underline{S}}} \mathrm{e}(\mathrm{t})$ le signal analytique associé au signal émis et $\underline{\underline{\underline{\underline{S}}}}(\mathrm{t})$. celui associé à un signal reçu. Supposons que $s_{\mathrm{r}}(\mathrm{t})$ soit simplement une réplique du signal émis, retardé d'une quantité $\tau(t)$ : 


$$
\begin{aligned}
& \underline{\underline{\underline{S}}} e(t)=u_{e} \exp \left(j \omega_{e} t\right) \\
& \underline{\underline{\underline{S}}} r(t)=u_{e} \exp \left(j \omega_{e}[t-\tau(t)]\right\}
\end{aligned}
$$

Pour simplifier nous considérons ici une amplitude constante pour le signal émis, comme c'est le cas pour un signal sinusoïdal. Dans ces conditions, l'argument du signal analytique émis est :

$$
\Phi_{\mathrm{e}}(\mathrm{t})=\omega_{\mathrm{e}} \mathrm{t}
$$

et l'argument de l'enveloppe complexe associée $\Delta \Phi_{\mathrm{e}}(\mathrm{t})$ est nul. La pulsation

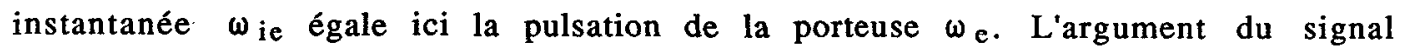
analytique retardé se déduit de (2) :

$$
\Phi_{r}(t)=\omega_{e}[t-\tau(t)]
$$

La pulsation instantanée correspondante, normalisée par division par $\omega_{\mathrm{e}}$, s'écrit :

$$
\omega_{i r}^{*}(t)=\frac{1}{\omega e} \frac{d \Phi_{r}(t)}{d t}=1-\frac{d \tau(t)}{d t}
$$

Quant à l'enveloppe complexe du signal retardé, son argument est :

$$
\Delta \Phi_{r}(t)=-\omega_{e} \tau(t)
$$

et sa pulsation instantanée, qui est la pulsation Doppler, est donnée sous sa forme normalisée par :

$$
\omega_{d}^{*}=\frac{1}{\omega e} \frac{d \Delta \Phi_{r}(t)}{d t}=-\frac{d \tau(t)}{d t}
$$

\section{3 - EFFET DOPPLER D'UNE CIBLE A VITESSE RADIALE VARIABLE}

Pour concrétiser les relations précédentes, considérons la situation décrite par la figure 1. Une cible $T$ se déplace selon un mouvement rectiligne uniforme à la vitesse v. Un transducteur unique, servant à la fois d'émetteur et de récepteur, est placé à la distance $r_{0}=r(t=0)$ de la trajectoire. L'analyse de cette situation montre que [5]:

$$
\begin{aligned}
& \tau(t)=\frac{2 v^{*}}{1-v^{*} 2}\left\{-v^{*} t+\left[t^{2}+r_{0}^{* 2} \frac{1-v^{* 2}}{v^{* 2}}\right]^{1 / 2}\right\} \\
& \omega_{d}^{*}(t)=\frac{2 v^{*}}{1-v^{* 2}}\left\{v^{*}-t\left[t^{2}+r_{0}^{* 2} \frac{1-v^{* 2}}{v^{* 2}}\right]^{-1 / 2}\right\}
\end{aligned}
$$

où $v^{*}$ et $r_{0}^{*}$ représentent respectivement les grandeurs normalisés $v / c$ et $r_{0} / c$. En remplaçant le temps $t$ par la grandeur adimensionnelle :

$$
\mathrm{t}^{*}=\mathrm{t} /\left[\frac{\mathrm{r}_{\mathrm{o}}^{*}}{\mathrm{v}}(1-\mathrm{v} * 2)^{1 / 2}\right]
$$




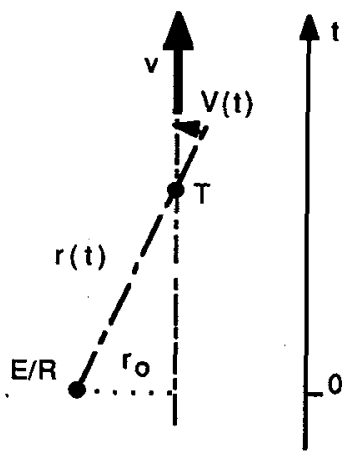

Fig. 1 : Position du transducteur

émetteur-récepteur par rapport à la cible $\mathrm{T}$ se déplaçant à la vitesse $\mathrm{v}$.

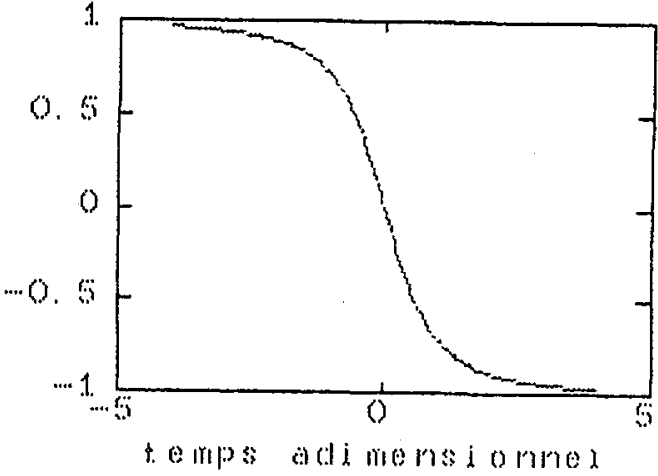

Fig. 2 : Représentation de la quantité $\omega_{d}^{*}(t) \frac{2 v^{*}}{1-v^{*} 2}$ en fonction de $t^{*}$.

on peut donner une représentation de l'expression (9) en représentant la quantité $\frac{1-v^{* 2}}{2 v^{*}} \omega_{d^{*}}\left(t^{*}\right)$ - figure 2 .

Comme il est possible d'accéder expérimentalement à l'enveloppe complexe du signal reçu par le récepteur [3], il est intéressant de prédire, par une simulation, le résultat qui serait obtenu dans une expérience réelle. C'est ce que nous avons fait en prenant pour cible une bulle qui s'élève, du fait de la poussée d'Archimède, à une vitesse de $30 \mathrm{~mm} / \mathrm{s}$ (bulle d'un rayon de l'ordre de $145 \mu \mathrm{m}$ ). Prenons $r_{0}=75 \mathrm{~mm}$ et supposons que l'amplitude de la pression incidente qui atteint la cible, ainsi que la pression captée par le récepteur après réflexion sur la cible, varient en $\mathbf{r}^{-1}$. Notons que dans les formules de la section précédente l'amplitude était considérée comme constante au cours du temps. La figure 3 montre l'évolution temporelle du module et de la phase (6) de l'enveloppe complexe associée au signal reçu, tandis que la figure 4 montre, dans le plan complexe, la courbe décrite par l'extrémité du vecteur associé à l'enveloppe complexe. Remarquons qu'une courbe simi laire a été obtenue lors d'une expérience réelle [6].
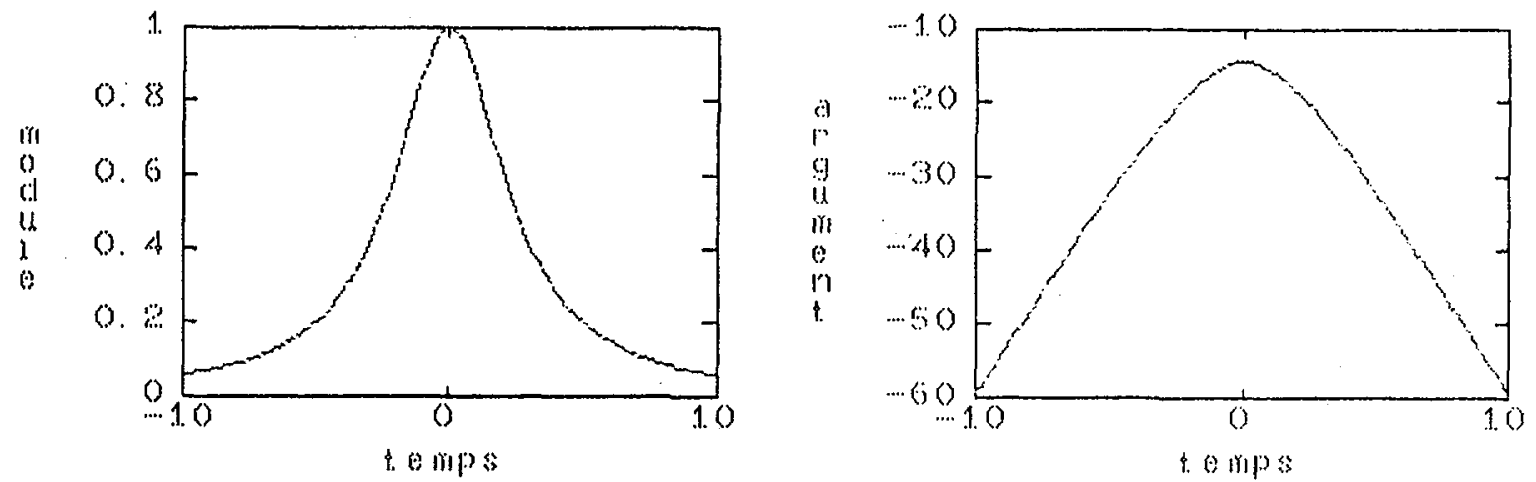

Fig. 3 : Evolution temporelle du module (à gauche) et de la phase (à droite) de l'enveloppe complexe associée au signal reçu par le transducteur récepteur. 


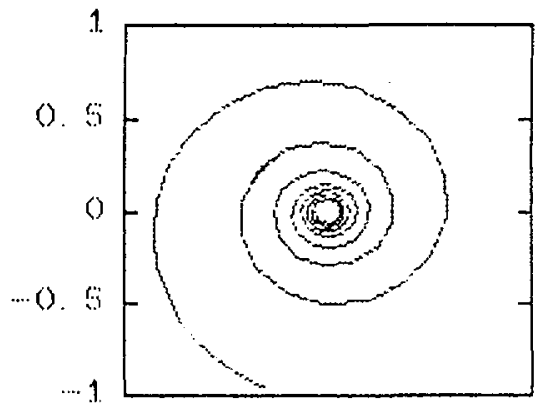

Fig. 4 : Représentation polaire du vecteur associé à l'enveloppe complexe.

\section{4 - CONCLUSION ET PERSPECTIVES}

Le présent travail montre que le signal analytique est un concept particulièrement adapté à la modélisation de l'effet Doppler quand celui-ci se traduit par une modulation à bande étroite de la porteuse. Il en est ainsi quand la variation de la vitesse de la cible est faible devant la célérité de l'onde acoustique.

La variation effective de l'expression qui a été simulée ici présente un intérêt direct: la description du champ incident. En effet la cible, si elle est suffisamment petite et si elle se comporte comme un reflecteur parfait (bille) agit comme un capteur ponctuel qui explore, au cours de son mouvement, le champ incident le long de sa trajectoire. Connaissant le champ incident il est alors possible d'étudier simultanément l'amplitude et la phase de la pression rayonnée par d'autres types de reflecteurs, comme par exemple des bulles.

\section{Bibliographie}

1 - F. DE COULON. Théorie et traitement des signaux (chaps.7 and 11), vol VI, Traité d'électricité. Lausanne, Switzerland, Presses Polytechniques Romandes, 1984.

2 - B. PICINBONO. Théorie des signaux et systèmes. Paris, Dunod, 1989.

3 - G. GIMENEZ., C. CACHARD., and D. VRAY D. Use of an analytic signal to model interaction between an acoustic wave and a moving target with a timedependent velocity. IEEE transactions on ultrasonics, ferroelectrics and frequency control, à paraître.

4 - C. CACHARD., G. GIMENEZ, D. VRAY. Ultrasonic Doppler device for measurement of time-depedent and space-dependent flow speed. IEEE Ultrasonics Symposium, 1988, Chicago-USA, vol. 2, p. 901-904.

5 - F. LE CHEVAliER. Principes de traitement des signaux radar et sonar. Paris, Masson, 1989, 271 p.

6 - G. GIMENEZ., C. CACHARD., F. LAKESTANI. Detection of stationary bubbles : experimental results. 10ème International Symposium on Non-linear Acoustics, 1984, Kobe-Japon, p. 89-93. 